\title{
Correlation of Ferritin and Transferrin Serum with hsCRP and F2-Isoprostane in Metabolic Syndrome
}

\author{
Waode Nurfina ${ }^{1,2^{*}}$, Irawan Yusuf ${ }^{2,3}$, and Mansyur Arif ${ }^{2,3}$ \\ 'Prodia Clinical Laboratory, Makassar, Indonesia \\ ${ }^{2}$ Post Graduate Program in Clinical Biochemistry, Hasanuddin University, Makassar, Indonesia \\ ${ }^{3}$ Faculty of Medicine Hasanuddin University, Makassar, Indonesia
}

\section{Abstract}

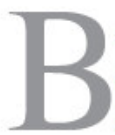

ACKGROUND: The low inflammatory state that accompanies the Metabolic Syndrome (MetS) associates with the overexpression of oxidative stress. Ferritin and Transferrin serum are often used to measure iron status and their concentrations are altered in several metabolic conditions. We hypothesized that concentration of Ferritin and Transferrin serum increase in Metabolic Syndrome (MetS) and correlate with the inflammation and oxidative stress.

METHODS: We studied 65 male metS patients, aged $43.26 \pm 7.16$ years. Iron metabolism was measured by concentration of Ferritin and Transferrin serums, while inflammatory and oxidative stress by high sensitivity C-reactive Protein (hsCRP) and F2-Isoprostane.

RESULTS: Concentration of Ferritin $315.70 \pm 188.63$ $\mathrm{ng} / \mathrm{L}$ and Transferrin $2.36 \pm 0.31 \mathrm{~g} / \mathrm{L}$ increased along with increasing components of metS. Concentration of Ferritin serum had a positive correlation with hsCRP $(\mathrm{r}=0.220)$ and F2-Isoprostane $(\mathrm{r}=0.023)$.

CONCLUSION: Serum concentration of Ferritin increased in the MetS and correlates with hsCRP and F2Isoprostane.

KEYWORDS: Metabolic Syndrome, Ferritin, Transferrin, hsCRP, F2-Isoprostane.

\section{Introduction}

The prevalence of MetS is rapidly increasing in developing as well as in developing countries including Indonesia. The main cause might be improvement in economic status along with the consumption of unhealthy diet (high in fat and cholesterol and less of fiber). Less of physical activity also contributes the occurrence of MetS. National Cholesterol Education Treatment Panel III (NCEP ATP III) identified there are 6 MetS components which are related to cardiovascular diseases, namely abdominal obesity, atherogenic dyslipidemia, high level of blood pressure, insulin resistance, proinflammatory status and prothrombotic status (1).

International Diabetes Federation (IDF) in 2005 (2) made a criteria of MetS: patient with central obesity (waist circumference male $\geq 90 \mathrm{~cm}$ and female $\geq 80$ $\mathrm{cm})$, plus two out of four following factors: Trigliceryde concentration $\geq 150 \mathrm{mg} / \mathrm{dL}$; HDL concentration male $<40$ $\mathrm{mg} / \mathrm{dL}$ and female $<50 \mathrm{mg} / \mathrm{dL}$; systolic blood pressure $\geq$ 130 or diastolic $\geq 85 \mathrm{mmHg}$; and fasting blood glucose concentration $\geq 100 \mathrm{mg} / \mathrm{dL}$ or has never been diagnosed with type 2 diabetes mellitus (3).

Several researches have been done on searching correlation or "cross talk" between insulin and iron metabolisms in type 2 diabetes mellitus patients but not yet on MetS (4). Meanwhile Moirand et al. (5), had proven that Hepatic iron overload (HIO) are related to insulin resistance, which was marked by insulin resistanceassociated HIO (IR-HIO), instead of being related to genetic factor of Hemochromatosis. 
The correlation of iron and insulin metabolisms is influenced by stress oxidative and inflammation status (4). The same thing had been proven by Festa, et al. (6) that there was a strong correlation of insulin resistance, inflammation, and coagulation in type 2 diabetes mellitus patients.

Central obesity is one kind of obesity which has a strong correlation with MetS, which is clinically marked by increase of waist circumference (1). Obesity contributes to increase of blood pressure, cholesterol serum, blood glucose as well as decrease of HDL concentration. The excess of adipose tissue on obese patients will result in the release of various factors such as Nonesterified Fatty Acid (NEFA), cytokines, Plasminogen Activator Inhibitor-1 (PAI-1) and other adipokines. The rise of CRP along with obesity will result in an excess of cytokines' release and the pro-inflammation states, meanwhile the rise of PAI-1 will contribute to the pro-thrombotic states (1). Obesity with MetS will increase the production of (radical oxidative stress) ROS from accumulated fat and thus increase the stress oxidative and affect the major organs such as liver, muscle and aorta (7).

Iron metabolism is a complex cycle of storage, usage, transport, elimination and reused. Iron metabolism in the body is a dynamic process; iron mostly absorbed in duodenum, the liver will then release Apo-Transferrin into bile duct and afterwards into the duodenum (8). The study by Sibille et al. in 1988 (9), was found that inflammation on sub-endothelial will result in the rise of Ferritin mRNA transcription in macrophage, while other study by Fernandez et al. (10) explained the increase concentration of Ferritin and Transferrin in type 2 diabetes mellitus is more due to inflammation rather than "iron overload". These findings supported the result of study by Zadeh (11) in haemodialysis that there is a correlation of hsCRP and Ferritin, in which concentration of Ferritin serum not solely regulated by iron concentration but also by inflammation and other factors.

The increase of plasma PAI- 1 in MetS is a result of the induction of TNF- $\alpha$, angiotensin II, interleukin- $1 \beta$, TGF- $\beta$, glucocorticoids and insulin. It explains the reason of the increasing PAI-1 in central obesity and diabetes mellitus, because in those two conditions the low level of chronic inflammation does occur. Festa, 2002 (6), has studied the increase of PAI-1 level has a positive correlation with the increase of Body Mass Index (BMI) and can be used as an independent predictor of type 2 diabetes mellitus. It is still not clear about the changes in iron metabolism in MetS, thus we examined our hypothesis that concentration of Ferritin and Transferrin serum will increase in MetS patients and they have a correlation with inflammation and oxidative stress.

\section{Methods}

\section{STUDY DESIGN AND SUBJECT RECRUITMENT}

Observational study was applied in this study on 74 male that fulfilled the MetS criteria of IDF 2005 (2), but 12 of them were excluded because of high level of SGPT, hsCRP and creatinin and thus only 65 samples were completely studied. The study protocol was approved for ethical clearance by the institutional review board of the Health Research Ethics Committee Faculty of Medicine, Hasanuddin University, Makassar, Indonesia (no. UH08120124). All participants filled in a written informed consent. For gathering the baseline data, each participant completed a self administered questionnaires covering medical history, exercise, usage of drug for lipid lowering, antioxidant or diabetes, smoking habits and alcohol intake. The questionnaires were checked by researcher at the screening phase. Subjects who were treated with antiinflammatory drugs in the last 3 weeks, or had chronic bleeding in the last 6 months, kidney dysfunction, liver dysfunction, fever and other acute inflammation were excluded from the study and asked to sign the informed consent prior to the commencement of the study.

All subjects were assessed after overnight fasting for a minimum of 10 hours. The parameters of anthropometric measurements (height, weight, waist circumference, and blood pressure) and biochemical variables (fasting blood glucose, creatinine, SGPT, hsCRP, Ferritin, F2-isoprostane) were measured in all subjects. Fasting serum samples were obtained and frozen at $-20^{\circ} \mathrm{C}$.

\section{ASSAY OF BIOCHEMICAL MARKERS}

The measurement of blood glucose was done by hexokinase method and the measurement of triglyceride by GPO PAP, the Dialine-manufactured reagent and Automatic Modular P800 (ROCHE) was used in both measurements. HDL cholesterol was obtained by homogeneous method Daichiimanufactured reagent, the level of hsCRP by solid phase chemiluminescent immunometric assay using Siemensmanufactured reagent and to obtain the serum level of Ferritin and Transferrin, immunochemiluminescence and immunoturbidimetry were used.

\section{STATISTICAL ANALYSIS}

Statistical analysis were performed by using SPSS 15 statistical software package. Univariate, bivariate and multivariate analysis were applied. Normal distribution of variables were assesed by applying Kolmogorov-Smirnov analysis. The relation of each parameter were analyzed by 
applying Pearson and Spearman's analysis with the alpha were set at 0.05 .

\section{Results}

Statistical analysis were performed on 65 subjects who fulfilled MetS criteria and other inclusive criterias. General description of the subject characteristics are shown in Table 1. Table 2 shows the significant correlation of Ferritin and MetS components, hsCRP as well as F2 Isoprostane. Table 3 shows the correlation of Ferritin serum with 2 and more components of MetS and indicating clearly that the more the components of MetS in subjects, the higher the Ferritin serum. Meanwhile from Figure 1 it is clear that correlation of Ferritin with the measurement values of inflammation (hsCRP) is stronger than that with stress oxidation state (F2-isoprostane) in MetS subjects.

Table 1. Basic Characteristics of the Study Subjects.

\begin{tabular}{lcccc}
\hline \multicolumn{1}{c}{ Variables } & Minimum & Maximum & Mean & SD \\
\hline Age (years) & 30.00 & 59.00 & 43.26 & 7.15 \\
\hline Clinical Variables & & & & \\
\hline Waist circumference $(\mathrm{cm})$ & 90.00 & 129.00 & 98.86 & 6.80 \\
\hline Biochemical Variables & & & \\
Ferritin (ng/L) & 19.30 & 920.00 & 315.70 & 188.63 \\
Transferrin $(\mathrm{g} / \mathrm{L})$ & 1.60 & 3.10 & 2.36 & 0.31 \\
FG $(\mathrm{mg} / \mathrm{dL})$ & 63.00 & 291.00 & 104.06 & 43.83 \\
HDL $(\mathrm{mg} / \mathrm{dL})$ & 27.00 & 73.00 & 40.41 & 8.91 \\
TG $(\mathrm{mg} / \mathrm{dL})$ & 114.00 & 830.00 & 261.45 & 131.53 \\
hsCRP $(\mathrm{mg} / \mathrm{L})$ & 0.20 & 10.00 & 2.04 & 2.00 \\
F2-isoprostane & 0.01 & 1.41 & 0.23 & 0.20 \\
\hline
\end{tabular}

Description: BMI = FG = Fasting Glucose, $\mathrm{TG}=$ triglyceride, hsCRP = high sensitivity C-Reactive Protein, HDL = High Density Lipoprotein sensitivity

Table 2. Correlation between variables.

\begin{tabular}{|c|c|c|c|c|}
\hline \multirow{2}{*}{ Variables } & \multicolumn{2}{|c|}{ Ferritin } & \multicolumn{2}{|c|}{ Transferrin } \\
\hline & $r$ & $\mathrm{p}$ & $r$ & $\mathrm{p}$ \\
\hline Waist circumference & 0.120 & 0.927 & 0.219 & 0.093 \\
\hline HDL & -0.125 & 0.343 & 0.042 & 0.750 \\
\hline TG & 0.220 & 0.092 & 0.284 & 0.028 \\
\hline FG & 0.022 & 0.867 & 0.193 & 0.139 \\
\hline SBP & 0.000 & 0.998 & 0.088 & 0.504 \\
\hline hsCRP & 0.220 & 0.094 & 0.093 & 0.474 \\
\hline F2-Isoprostane & 0.023 & 0.860 & 0.163 & 0.205 \\
\hline
\end{tabular}

Description: FG = Fasting Glucose, $\mathrm{SBP}=$ Systolic Blood Pressure, $\mathrm{TG}=$ triglyceride, $\mathrm{hsCRP}=$ high sensitivity $\mathrm{C}-$ Reactive Protein, $\mathrm{HDL}=$ High Density Lipoprotein cholesterol 
Table 3. Independent t-test MetS Components and Ferritin

\begin{tabular}{lcccc}
\hline Variables & $\begin{array}{c}\text { 2 components MetS } \\
\text { mean } \pm \text { SD }\end{array}$ & $\begin{array}{c}3 \text { components Mets } \\
\text { mean } \pm \text { SD }\end{array}$ & $\begin{array}{c}\text { 4 components Mets } \\
\text { mean } \pm \text { SD }\end{array}$ & $\begin{array}{c}\text { 5 components MetS } \\
\text { mean } \pm \text { SD }\end{array}$ \\
\hline $\mathrm{N}$ (male) & 10 & 31 & 15 & 9 \\
Transferrin & $2.32 \pm 0.28$ & $2.34 \pm 0.28$ & $2.34 \pm 0.35$ & $2.53 \pm 0.33$ \\
Ferritin & $288 \pm 158$ & $302 \pm 208$ & $330 \pm 198$ & $398 \pm 246$ \\
\hline
\end{tabular}
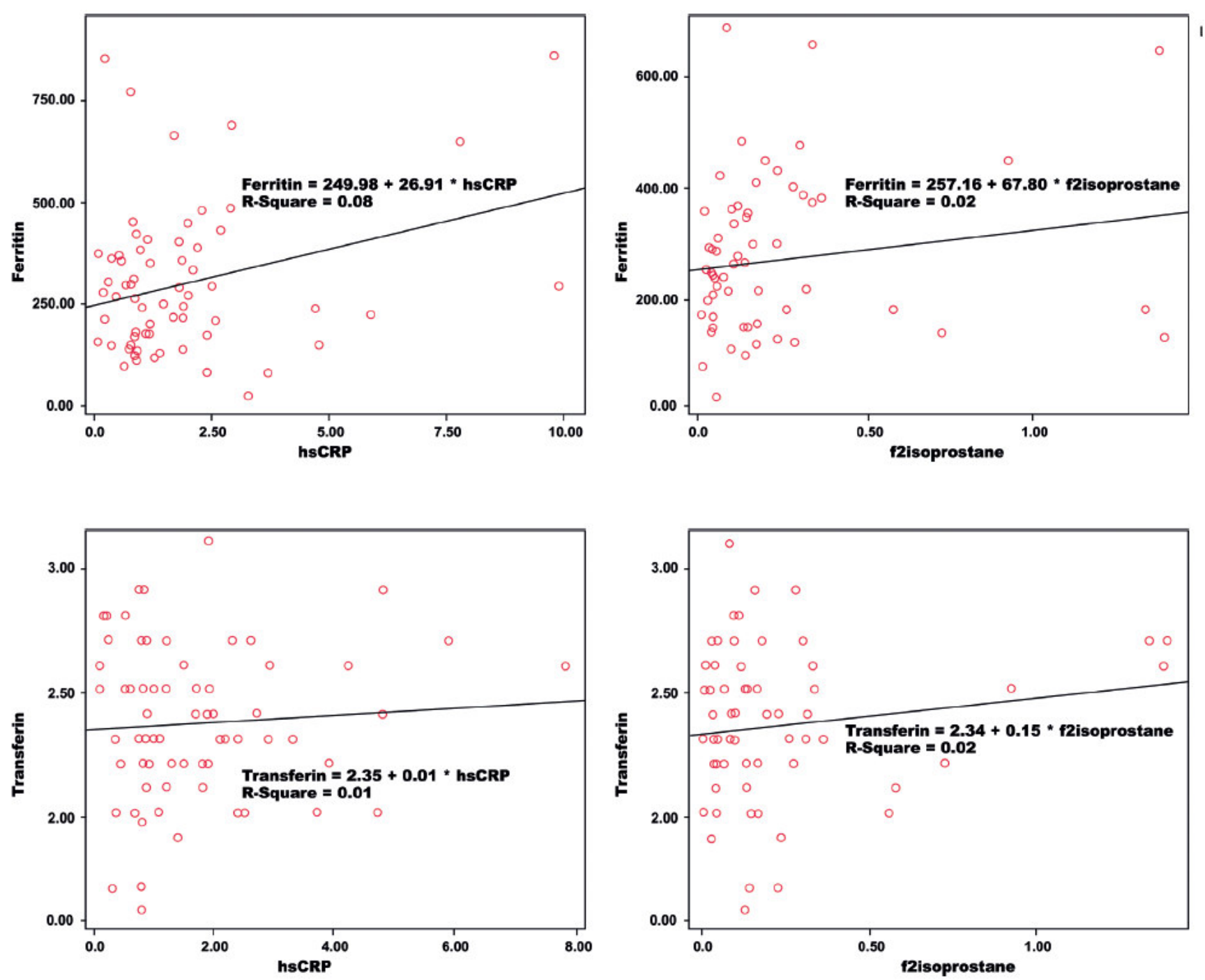

Figure 1. Correlation of Ferritin, Transferrin and hsCRP as well as F2 - Isoprostane. 


\section{Discussions}

This study showed there was a change in iron metabolism (Ferritin and Transferrin) in MetS subjects compare with the normal value as written on the kit insert. The concentration of Ferritin and Transferrin serum in the subjects were at the upper normal limit (normal value Ferritin $=28-365 \mathrm{ng} / \mathrm{mL}$; Transferrin $=2.24-4.06 \mathrm{~g} / \mathrm{L}$ ) and thus below the concentration on hemocromatosis. To analyze the correlation of MetS components and Ferritin as well as Transferrin, Partial correlation analysis were used. Table 2 shows that MetS components has a weak positive correlation with iron metabolism (except Ferritin has a negative correlation with HDL). This finding supports the study of Istvan S (12), that not only the iron storage (Ferritin) but also its protein transporter (Transferrin) has a role in insulin resistance and MetS. Our data showed a positive correlation of Transferrin and Triglyceride which explains the role of Transferrin in lipolysis process in adipose tissue, thus increases the concentration of free fatty acid in liver and muscle. Adipose tissues express the Transferrin receptor and therefore can bind the iron boundTransferrin by endocytosis process. In MetS condition in which there is an increase in adipose tissues indirectly that will increase the cellular iron uptake and in turn accelerate the lipolysis process by iron pro-oxidized effect (13).

When Ferritin and Transferrin were compared with two and more components of MetS as shown in Table 3, it is clear that the concentration of Ferritin and Transferrin serum increased along with the increase number of MetS components, which is in accordance with the finding by Wrede (14).

Ferritin is an acute phase protein; its concentration will rise in inflammation state as seen in MetS which is a low level of chronic inflammation state. As can be seen in Figure 1, that the concentration of Ferritin serum increases along with concentration of hsCRP $(<10 \mathrm{mg} / \mathrm{dL}=$ chronic inflammation). Proinflammatory cytokines (TNF- $\alpha$, IL-1 and $\mathrm{IL}-6$ ) increases the expression of divalent metal transporter 1 (DMT-1) in the macrophage, and thus induces the expression of Ferritin and reduces the expression of Ferroportin. Meanwhile, cytokines will stimulate the uptake of iron bound-Transferrin into the macrophages by Transferrin receptor. In the inflammation state, activated macrophage will release Apolactoferin which has ironaffinity 4-times stronger than that in Transferrin and it will deliver iron into cell faster and therefore increase the Ferritin synthesis, and this explains why in inflammation state there is an increase of Ferritin but not Transferrin.

Iron has a strong correlation with stress oxidative state by Fenton reaction in producing free radicals such as hydroxide and superoxide anion which has a role in lipid peroxidation. However in this study the correlation of Ferritin serum and F2-isoprostane is weak and it might be due to the direct role of oxidative stress in releasing iron from Ferritin and not due its synthesis. From the study of Fernandez (4), there was a strong correlation of iron and oxidative stress in type 2 diabetes mellitus, Transferrin in those subjects might be glycated and thus decrease its affinity to free iron $\left(\mathrm{Fe}^{+}\right)$thus stimulated the synthesis of Ferritin.Another explanation of this finding is that the source of free radicals in MetS not only from iron metabolism but also from hyperglycemic or hypertension state, in other words there exists a compensation mechanism between oxidative stress and iron (15).

\section{Conclusions}

In conclusion, the study has shown that iron metabolism has a positive correlation with two or more components of MetS, as the free iron must be in limited amount in blood circulation and thus Ferritin synthesis will increase in MetS in which there is a process such as chronic inflammation and oxidative stress. However the concentration of Transferrin has not shown the same thing and it might be due to there is another kind of iron transportation such as Apolactoferrin released from activated macrophage in inflammation state. Therefore further studies are still required to investigate other factors such as those that have a role in iron metabolism such as Hepcidin, Apolactoferin, etc. to completely elucidate the pathomechanism in the role of iron metabolism in MetS. It is also needed to have a comparison study of non Obese and Obese MetS subjects to find out the changes of iron metabolism as well as to determine a cut off point of Ferritin and Transferrin, thus can be used to assess MetS and its complications.

\section{Acknowledgements:}

We thank the Prodia Education and Research Institute for the invaluable support given to this study. 


\section{References:}

1. Grundy S, Brewer B, Cleeman Jl, Smith S, and Lenfant C. Definition of Metabolic Syndrome, Report of National Heart, Lung and Blood Institute/American Heart Association Conference on Scientific Issues Related to Definition, Circulation 2004; 109: 433-8

2. International Diabetes Federation. The IDF Consensus Worldwide Definition of The Metabolic Syndrome 2005

3. Rana JS. Cardiovascular Metabolic Syndrom-an Interplay of Obesity, Inflammation, Diabetes and Coronary Heart Disease. Diabetes Obes Metab 2007; 9: 218-32

4. Jose Manual F, Abel Lopez Bermejo, Wifredo Richart. Cross talk Between Iron Metabolisme and Diabetes, Diabetes Care 2002; 51: 2348-2354

5. Moirand R, Mortaji A, Lore' al O, Paillard F, Brissot P, Deugnier $Y$. A New Syndrome of Liver Iron Overload with Normal Transferrin Saturation. Lancet 1997; 349: 95-7

6. Festa A, Anthony J.G Hanley, Russel P. Tracy, Ralph D'Agostino, $\mathrm{Jr}$, dan Steven M. Haffner. Elevated Level of Acute-phase Proteins and Plasminogen Activator Inhibitor-1 Predict the Development of Type 2 Diabetes, Circulation 2002; 5; $1131-7$

7. Furukawa $S$, Fujita $T$, Shimabukuro M, Iwaki M, Yukio $T$, Nakajima $Y$, et al. Increased Oxidative Stress in Obesity and its Impact on Metabolic Syndrome, J. Clin. Invest. 2004; 114: 1752-61

8. Andrews NC. Disorders of Iron Metabolism. New England Journal of Medicine 1999; 341: 1986-94
9. Sibille JC, Kondo G and Aisen P. Interaction between isolated hepatocytes and Kupffer cells in iron metabolism; a possible role for ferritin as an iron carrier protein. Hepatology 1988; 8: 296-301

10. Ferna'ndez-Real JM, Ricart W, Arroyo E, Balanc,a R, Casamitjana R CabreroD. Serum Ferritin as a Component of The Insulin Resistance Syndrome. Diabetes Care1998; 21: $62-8$

11. Zadeh, $\mathrm{K}$, et al. Association Between Serum Ferritin and Measure of Inflammation, Nutrition, and Iron in Hemodialysis Patients. Nephrology Dialysis Transplantation 2004; 19: 141-9

12. Istvan SV, Balkau B, Kettaneh A. Ferritin and Transferrin are Associated with metabolic syndrome abnormalities and their change over time in general population. Diabetes Care 2007; 30/7: 1795-1801

13. Fumeron F, Frank Pinn, Fatti Dris, Beverly Balkau, Jean Tichet, Michel Marre, et al. Ferritin and Transferrin are Both Predictive of the Onset of Hyperglycemia in Men and Women over 3 years. Diabetes Care 2006; 29: 2090-4

14. Wrede CE. Association Between Serum Ferritin and The Insulin Resistance Syndrome in a Representative Population. European Journal of Endocrinology 2006; 154: 333-40

15. Juckett MB, Balla J, Balla G et al. Ferritin Protects Endothelial Cells From Oxidized Low Density Lipoprotein in Vivo. American Journal of Pathology 1995; 147: 782-9 